\title{
Attitude Stabilization of a Quadrotor Helicopter Using Brushless Motors
}

\author{
Xiaorong Wang \\ Early-childhood Teachers Normal College \\ Xi'an University of Arts and Science \\ Xi'an, China \\ xrongwang@126.com \\ Suoli Guo \\ Xi'an Research Institute of Hi-Tech \\ Xi'an, China \\ e-mail: analog2k4@126.com
}

\author{
Mengxuan Guo \\ School of Automation and Information Engineering \\ Xi'an University of Technology \\ Xi'an, China \\ e-mail: 350538395@qq.com
}

\author{
Lining Tan \\ Xi'an Research Institute of Hi-Tech \\ Xi'an, China \\ e-mail: oldbat@126.com
}

\begin{abstract}
Control system design of quadrotor helicopters with accurate actuator modeling is an important practical design problem that many previous works did not consider. In particular, most actuators of such vehicles are brushless motors, which are hardly controlled without accurate dynamical models. In order to improve the performance of attitude stabilization control system, a new control strategy is proposed in this paper. This control strategy is based on the nonlinear modeling of brushless motors. To reduce the complexity of traditional nonlinear Volterra models, we propose the use of Hammerstein series models. Furthermore a method for identification of such model from observed input-output data is presented. As models of four brushless motor have been obtained, a backstepping attitude controller is designed to stabilize the quadrotor helicopter. Flight experiments show that the proposed control strategy can achieve higher performance than original ones.
\end{abstract}

Keywords- Quadrotor helicopter; Attitude control; Nonlinear system; Hammerstein series model; Backstepping

\section{INTRODUCTION}

Quadrotor unmanned helicopter is a system consisting of two pairs of counter-rotating rotors attached at each corner of a cross frame. It has two main advantages over traditional one main rotor helicopters. First, the mechanical construction is simpler because of the elimination of complex mechanical control linkages for rotor actuation. Second, the use of four rotors leads to the smaller individual rotors than the main rotor on a traditional helicopter for certain airframe size, which permitting flights with reduced risk of damage to the vehicles, operators and surroundings. For these reasons, the quadrotor helicopter and its control scheme have received increased attention in recent years.

Nevertheless, quadrotors have high nonlinear behavior and they are typical under-actuated systems. Therefore, the design of a controller for these vehicles is very difficult and is still a challenge for researchers. Many efforts have been made to control quadrotor helicopters. The dynamical model of the quadrotor helicopters has been studied in ${ }^{[1]}$. $\mathrm{In}^{[2]}$, a modification on this dynamical model is made and backstepping controller for quadrotors' trajectory tracking is designed. To obtain stability in flying the quadrotor, a sliding mode controller for a fully-actuated subsystem of a quadrotor is designed in ${ }^{[3]}$. In order to achieve desirable attitude and position control in the presence of disturbance such as gust, ground effects, inaccurate measurements and the bias between the geometric centre and its centre of gravity, disturbance observer or estimator is designed and combined with other well-known controller ${ }^{[4-6]}$. For the same reason, a robust adaptive controller to achieve both adaptation to unknown payloads and robustness to disturbance is presented in ${ }^{[7]}$

However, for the controller design issue, nonlinear dynamics of brushless motor are seldom considered. In ${ }^{[8-9]}$, the important role of dynamics of drive system is well recognized and the linear models of motor are taken account into the controller design. But as the brushless motor is a typical nonlinear system ${ }^{[10]}$, the simple linear model can not well characterize their dynamical behavior.

In this paper, an accurate but simple nonlinear model of brushless motor is considered in the stabilization control of a quadrotor helicopter. To reduce the complexity of traditional nonlinear Volterra models, we propose the use of the Hammerstein series models. By taking the nonlinear models of brushless motor into account, a backstepping attitude controller is designed to stabilize the quadrotor helicopter. Flight experiments prove that, compared to past control strategies which have not considered the motor dynamics, the proposed one can achieve autonomous flight with higher performance.

\section{PRELIMINARY}

\section{A. Quadrotor Modeling}

Quadrotor helicopter, as shown in Fig .1, has four rotors to generate the thrust forces $T_{j}$ and rolling moments $\mathrm{M}_{\mathrm{j}}, \mathrm{j}=1,2,3,4$, . Let $\mathrm{B}=\left\{\mathrm{O}_{\mathrm{b}} \mathrm{x}_{\mathrm{b}} \mathrm{y}_{\mathrm{b}} \mathrm{z}_{\mathrm{b}}\right\}$ denote a body fixed frame linked to the vehicle whose origin $\mathrm{O}$ is at the center of mass of the quadrotor and $\mathrm{E}=\left\{\mathrm{O}_{\mathrm{e}} \mathrm{x}_{\mathrm{e}} \mathrm{y}_{\mathrm{e}} \mathrm{Z}_{\mathrm{e}}\right\}$ an inertial frame fixed with respect to the earth. The attitude of 
quadrotor is represented by three Euler angles $\Theta=(\phi \theta \psi)^{\mathrm{T}}$ and the body angular velocities by $\omega=(\mathrm{p} \mathrm{q} \mathrm{r})^{\mathrm{T}}$.

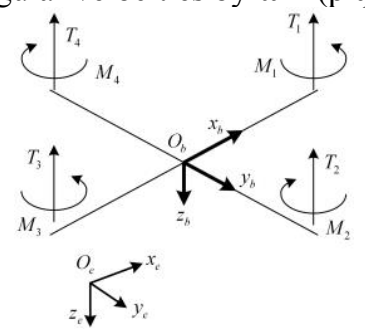

Figure 1. Quadrotor helicopter scheme

The dynamic equation of attitude motion is

$$
J \boldsymbol{Q} \&=\tau-\boldsymbol{\tau} \times J \boldsymbol{\omega}
$$

where $\tau=\left(\begin{array}{lll}\tau_{\mathrm{x}} & \tau_{\mathrm{y}} & \tau_{\mathrm{z}}\end{array}\right)^{\mathrm{T}}$ is the total torques applied to the center of mass of the quadrotor, $\mathrm{J}$ is the inertia moments matrix. By assuming the quadrotor is symmetrical, the inertia moments matrix can be written as

$$
J=\left[\begin{array}{ccc}
I_{x x} & 0 & 0 \\
0 & I_{y y} & 0 \\
0 & 0 & I_{z z}
\end{array}\right]
$$

The major goal of attitude controller design is to generate $\tau$ to make $\omega$ tracking the desired angle rates.

Thrust force of a rotor, the resultant of the vertical forces acting on all the blade elements, can be written as

$$
T=C_{T} \rho A(\Omega R)^{2}
$$

where $C_{T}$ is the thrust coefficient, $\rho$ is the air density, $A$ is the propeller disk area, $\Omega$ is the propeller angular rate and $R$ is rotor radius. Similarly, rolling moment of a rotor can be written as

$$
M=C_{M} \rho A(\Omega R)^{2} R
$$

where $C_{M}$ is the rolling moment coefficient. Then the force $\mathbf{F}$ and torque $\boldsymbol{\tau}$ produced by the drive system are

$$
\begin{aligned}
& \mathbf{F}=\left[\begin{array}{c}
0 \\
0 \\
T_{1}+T_{2}+T_{3}+T_{4}
\end{array}\right] \\
& \boldsymbol{\tau}=\left[\begin{array}{c}
l\left(T_{2}-T_{4}\right) \\
l\left(T_{3}-T_{1}\right) \\
M_{1}-M_{2}+M_{3}-M_{4}
\end{array}\right]
\end{aligned}
$$

where 1 is the horizontal distance from propeller centre to centre of gravity.

For given desired angle rates, the required total torque $\boldsymbol{\tau}$ can be obtained by solving Eq. (1). Then given $\mathbf{F}$, the desired rotor angular rate $\Omega$ can be obtained by solving Eq. (3) - Eq. (6).

\section{B. Hammerstein Series Model}

Hammerstein series model is a subclass of widely used Volterra series models [11]. The major advantage of this kind of models is the trade-off between parametric complexity and model generalization ability [12]. The second order case of Hammerstein series model is used in this paper to model the brushless motor, and it has the form

$$
y(t)=\sum_{k=0}^{\infty} g_{1}(k) u(t-k)+\sum_{k=0}^{\infty} g_{2}(k) u^{2}(t-k)
$$

where $u(t)$ and $y(t)$ is the input and output of the system, respectively. Functions $\left\{g_{n}(\tau)\right\}, n=1,2$ describe the linear and quadratic response of the system, and are called the Hammerstein linear and quadratic kernel, respectively.

In practice, we consider the second Hammerstein series model has finite memory length $M$ and the output is disturbed by noise, as can be seen in Fig .2, then Eq. (7) can be rewritten as

$$
y(t)=\sum_{k=0}^{M-1} g_{1}(k) u(t-k)+\sum_{k=0}^{M-1} g_{2}(k) u^{2}(t-k)+v(t)
$$

where $v(t)$ is a zero-mean additional white Gaussian noise, and independent from the input $u(t)$ and the output $y(t)$.

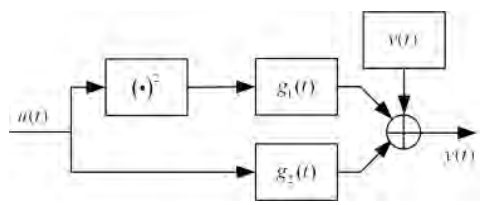

Figure 2. Second order Hammerstein series model scheme

\section{MODELING OF THE BRUSHLESS Motor}

\section{A. Estimation of Hammerstein Kernels}

The excitation of brushless motor is a Pulse Width Modulated (PWM) signal sent to the motor control chip in a 255-step duty cycle. By recording the input PWM signal and the motor's angular rate, a data set with $N$ pairs inputoutput data can be obtained.

Let $Z^{N} @\{u(1), y(1), \ldots, u(N), y(N)\}$ be the observed data set, and for given memory length $m$, Eq. (8) can be rewritten in vector form

$$
\mathbf{y}=\boldsymbol{\Phi} \boldsymbol{\theta}+\mathbf{v}
$$

with

$$
\begin{aligned}
& \mathbf{y}=(y(1), \ldots, y(N))^{T} \\
& \mathbf{v}=(v(1), \ldots, v(N))^{T} \\
& \boldsymbol{\Phi}=\left(\begin{array}{ll}
\mathbf{X}_{1} & \mathbf{X}_{2}
\end{array}\right) \\
& \mathbf{X}_{k}=\left[\begin{array}{cccc}
x(1)^{k} & x(0)^{k} & \mathrm{~L} & x(1-m)^{k} \\
x(2)^{k} & x(1)^{k} & \mathrm{~L} & x(2-m)^{k} \\
\mathrm{M} & \mathrm{M} & \mathrm{O} & \mathrm{M} \\
x(N)^{k} & x(N-1)^{k} & \mathrm{~L} & x(N-m)^{k}
\end{array}\right] \\
& \boldsymbol{\theta}=\left(\begin{array}{ll}
\mathbf{g}_{1} & \mathbf{g}_{2}
\end{array}\right)^{T} \\
& \mathbf{g}_{k}=\left(\begin{array}{llll}
g_{k}(0) & g_{k}(1) & \mathrm{L} & \left.g_{k}(m)\right)^{T}
\end{array}\right.
\end{aligned}
$$

where $\boldsymbol{\Phi}$ is a $N \times 2(m+1)$ block matrix with elements $\mathbf{X}_{k}$ and $\boldsymbol{\theta}$ is the $2(m+1)$-vector valued parameter with elements $\mathbf{g}_{k}(\tau), \tau=0, \ldots, m$, and $k=1,2$.

The Least-squares estimate (LES) of $\boldsymbol{\theta}$ is then obtained from

$$
\hat{\boldsymbol{\theta}}=\left(\boldsymbol{\Phi}^{T} \boldsymbol{\Phi}\right)^{-1} \boldsymbol{\Phi}^{T} \mathbf{y}
$$

provided the inverse exists. Then nonlinear behaviors of the brushless motor can be accurately characterized by the second order Hammerstein series model. Given the desired rotor angular rate $\Omega$, the corresponding control 
signal can be obtained by solving Eq. (9) with estimated $\hat{\boldsymbol{\theta}}$. It is remarkable that Eq. (9) do not need the past values of rotor's angular rate, which make the open-loop control of drive system can be achieved.

\section{B. Excitation Design}

As our goal is to stabilize the attitude of quadrotor near hovering, the equilibrium state of brushless motor is selected in 200 PWM units. This state is obtained by real flight experiments, and when the quadrotor is hovering, each motor input is closed to 200 PWM units.

In identification experiment, a random multi-sine signal will be used. This excitation is a broadband, periodic signal:

$$
u(t)=A_{0}+\sum_{k=1}^{K} A_{k} \cos \left(2 \pi \frac{k f_{\max }}{K} t+\varphi_{k}\right)
$$

where $f_{\max }$ is the maximum frequency of the excitation and $K$ is the number of frequency components. The phases $\left\{\varphi_{k}\right\}$ are independent uniformly distributed random variables on $[0,2 \pi)$, such that $E\left\{e^{j \varphi_{k}}\right\}=0 . A_{0}$ is the equilibrium state of system that will be excited. The amplitudes $\left\{A_{k}\right\}$ are real independent, identically, distributed random variables. Then the higher order moments of $A_{k}$ should remain bounded for any finite order. These signals can cancel leakage effects due to their periodic property. They also provide a prefect cut-off of power spectrum, which can avoid aliasing.

\section{Modeling Procedures}

The complete modeling procedure for brushless motor using second order Hammerstein series model is outlined as below:

Step 1: Choose $f_{\max }$ and $K$, so that the interesting behaviors of the system under study are in the frequency range $\left[f_{\max } / K, f_{\max }\right]$.

Step 2: Determine the sampling frequency $f_{s}$ to avoid aliasing effects.

Step 3: Generate independent random phase $\left\{\varphi_{k}\right\}$ and required amplitudes $\left\{A_{k}\right\}$, construct the identification excitation signal using Eq. (17)..

Step 4: Apply the excitation to the brushless motor system and collect the time series record of steady-state rotor angular rate $y(t)$ corresponding to $u(t)$.

Step 5: Estimate Hammerstein kernels using Eq. (16).

As the estimation of Hammerstein kernels $\hat{\boldsymbol{\theta}}$ are obtained, for given desired rotor angular rate, the openloop control signal can be obtained by solving Eq. (9) with past $m-1$ control signals.

\section{BACKSTEPPING CONTROLLER DESIGN}

\section{A. Modeling for Control}

The model Eq. (1) describes the differential equation of attitude motion of the quadrotor helicopter. In order to design a backstepping controller for this model, it can be rewritten in state-space form $\mathbf{k}=f(\mathbf{X}, \mathbf{U})$ with $\mathbf{U}$ inputs vector and $\mathbf{X}$ state vector chosen as

$$
\begin{gathered}
\mathbf{X}=\left(x_{1}, x_{2}, x_{3}, x_{4}, x_{5}, x_{6}\right)^{T} \\
=(\phi, \phi, \theta, \phi, \psi, \psi \&)^{T} \\
\quad \mathbf{U}=\left(U_{1}, U_{2}, U_{3}\right)^{T}=\boldsymbol{\tau}
\end{gathered}
$$

The transformation matrix between the rates of change of attitude $(\phi, \&, \& \&)$ and the body angular velocities $(p, q, r)$ can be considered as unity matrix as the perturbations in stability flight are small. This mean one can write $(\phi, \phi, 4 \&) \approx(p, q, r)$. From Eq. (1), Eq. (2), Eq. (18) and Eq. (19) we obtain the model for control design as

$$
\left\{\begin{array}{l}
\mathbb{\&}=x_{2} \\
\&=\left(U_{1}-\left(I_{z z}-I_{y y}\right) x_{4} x_{6}\right) / I_{x x} \\
\&=x_{4} \\
\&=\left(U_{2}-\left(I_{x x}-I_{z z}\right) x_{2} x_{6}\right) / I_{y y} \\
\&=x_{6} \\
\&=\left(U_{1}-\left(I_{y y}-I_{x x}\right) x_{2} x_{4}\right) / I_{z z}
\end{array}\right.
$$

\section{B. Backstepping Controller Design}

Backstepping method is a recursive procedure for systematically selecting the control Lyapunov functions that allows the design of adaptive controllers for a class of nonlinear processes. Using the backstepping method, one can synthesize the control law forcing the system to follow the desired trajectory [13].

For given desired roll angular $x_{1}^{d}$ and current $x_{1}$, the tracking error can be obtained by

$$
e_{1}=x_{1}^{d}-x_{1}
$$

By using the Lyapunov theorem, we consider the Lyapunov function $V\left(e_{1}\right)$ positive definite and its time derivative negative semi-definite:

$$
\begin{aligned}
& V\left(e_{1}\right)=\frac{1}{2} e_{1}^{2} \\
& \left.I \& e_{1}\right)=e_{1}\left(\&-x_{2}\right)
\end{aligned}
$$

The stabilization of $e_{1}$ can be guaranteed by letting

$$
x_{2}=\alpha_{1} e_{1}+\not{R}
$$

with $\alpha_{1}>0$. And then Eq. (22) is $\left.\& e_{1}\right)=-\alpha_{1} e_{1}^{2} \leq 0$. Let Eq. (24) be the desired roll angular velocity, we can obtain the tracking error of current $x_{2}$ :

$$
e_{2}=\alpha_{1} e_{1}+x_{2}
$$

Then considering the augmented Lyapunov function

$$
V\left(e_{1}, e_{2}\right)=\frac{1}{2}\left(e_{1}^{2}+e_{2}^{2}\right)
$$

and its time derivative

$$
\begin{aligned}
\&\left(e_{1}, e_{2}\right) & =e_{1} \&+e_{2}\left(\alpha_{1} \&+-\&\right) \\
& =e_{2}\left(-U_{1}+\left(I_{z z}-I_{y y}\right) x_{4} x_{6}\right) / I_{x x} \\
& -\alpha_{1}^{2} e_{1} e_{2}+e_{2}-\alpha_{1} e_{1}^{2}
\end{aligned}
$$

In attitude stabilization control, we always expect the angular accelerate is zero, i.e. $=0, i=1,3,5$. Then the control input $\mathrm{U}_{1}$ can be extracted by satisfying $\&\left(e_{1}, e_{2}\right) \leq 0$ : 


$$
U_{1}=\left(I_{z z}-I_{y y}\right) x_{4} x_{6}+I_{x x}\left(\alpha_{2} e_{2}-\alpha_{1}^{2} e_{1}\right)
$$

with $\alpha_{1}>0$. Similarly, $U_{2}$ and $U_{3}$ can be extracted with same method:

$$
\begin{gathered}
U_{2}=\left(I_{x x}-I_{z z}\right) x_{2} x_{6}+I_{y y}\left(\alpha_{4} e_{4}-\alpha_{3}^{2} e_{3}\right) \\
U_{3}=\left(I_{y y}-I_{x x}\right) x_{2} x_{4}+I_{z z}\left(\alpha_{6} e_{6}-\alpha_{5}^{2} e_{5}\right)
\end{gathered}
$$

with

$$
\left\{\begin{array}{l}
e_{3}=x_{3}^{d}-x_{3} \\
e_{4}=\alpha_{3} e_{3}+\frac{d}{3}-x_{4} \\
e_{5}=x_{5}^{d}-x_{5} \\
e_{6}=\alpha_{5} e_{5}+x_{5}^{d}-x_{6}
\end{array}\right.
$$

\section{EXPERIMENTS AND DISCUSSION}

\section{A. Nonlinear Model of Brushless Motor}

In the identification experiments, a random multi-sine excitation with 32 frequency components is used with $\mathrm{f}_{\max }=2.5 \mathrm{~Hz}, \mathrm{~A}_{\mathrm{k}}=25, \mathrm{~A}_{0}=200$ and sampling frequency $\mathrm{f}_{\mathrm{s}}=2.5 \mathrm{~Hz}$. Then the modeling procedure is carried out follow steps described in section 3.3.

In order to check the "goodness" of the model, the model error (in $\mathrm{dB}$ ) between actual outputs and model outputs is defined as

$$
q(t)=10 \log _{10}\left(\frac{|y(t)-\hat{y}(t)|}{|y(t)|}\right)
$$

where $y(t)$ and $\hat{y}(t)$ are the actual and model outputs, respectively. Besides, to validate the different model outcomes, a total fitness will be used:

$$
\text { fit }=\left(1-\frac{\sqrt{\sum_{t=1}^{N}(y(t)-\hat{y}(t))^{2}}}{\sqrt{\sum_{t=1}^{N}(y(t)-\bar{y}(t))^{2}}}\right) \times 100 \%
$$

which is a measure of how much better the model describes the system compared to the mean of the output..

A comparison of behaviors using linear model and second order Hammerstein model is also studied. The experiments results can be seen in Fig .3 - Fig .6 and Table. 1.

TABLE I. FITNESS COMPARISON OF LINEAR AND NONLINEAR MODELS

\begin{tabular}{|c|c|c|}
\hline Models & Linear model & Nonlinear model \\
\hline Fitness & $68.53 \%$ & $87.03 \%$ \\
\hline
\end{tabular}

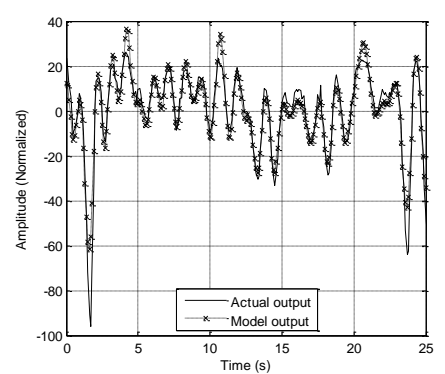

Figure 3. Linear model output tracking curve

Comparing the model performance shown in Fig .3 Fig .6, it can be seen that the proposed nonlinear model has more accurate tracking ability. As the linear model has a bad tracking performance and high model error, control signals extracted from such model can not drive brushless motors for the desired angular rates. It will make the control issue of quadrotor helicopters more difficult. Thus we strongly recommend the use of second order Hammerstein model of brushless motors, which not only has high accuracy, but also with low model complexity.

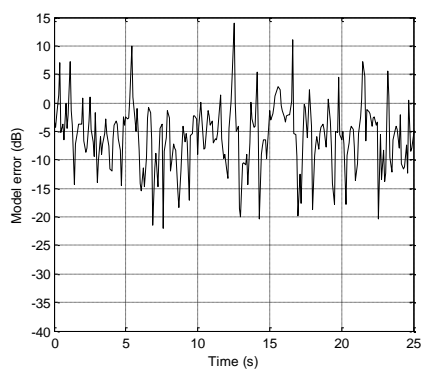

Figure 4. Linear model error

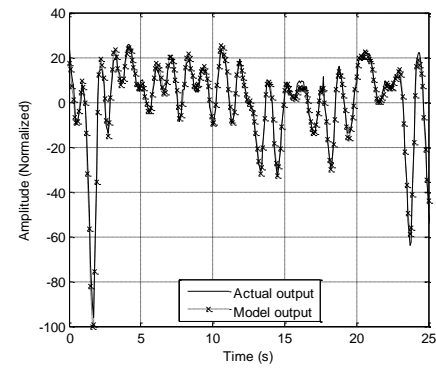

Figure 5. Nonlinear model output tracking curve

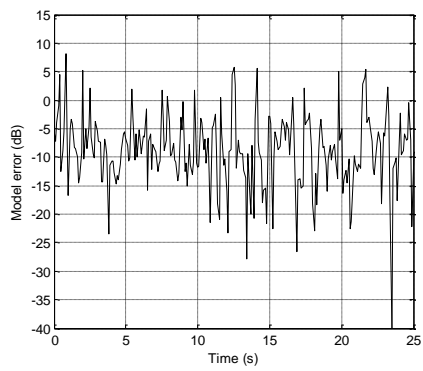

Figure 6. Nonlinear model error

\section{B. Attitude Stabilization of Real System}

In order to validate the control strategy developed in this paper, we implemented the controller on a STM32 processor running at $72 \mathrm{MHz}$. We performed several experiments on the real system, where the task is to stabilize the attitude of the quadrotor helicopter. The altitude was then fixed by the operator.

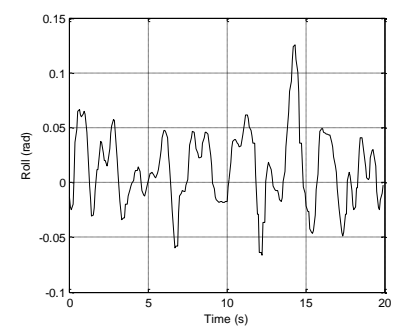

Figure 7. Performance of conventional backstepping controller to stabilize the quadrotor and maintain the roll angle to zero 


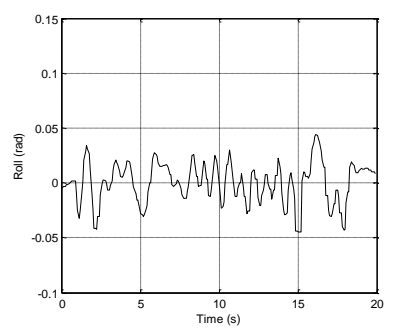

Figure 8. Performance of proposed controller to stabilize the quadrotor and maintain the roll angle to zero

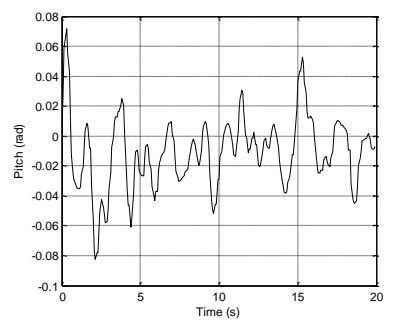

Figure 9. Performance of conventional backstepping controller to stabilize the quadrotor and maintain the pitch angle to zero

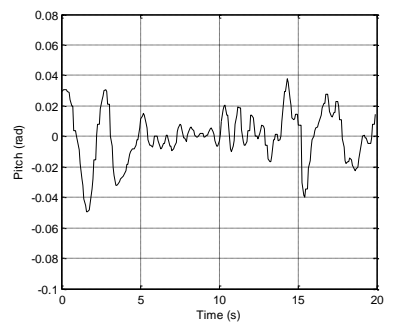

Figure 10. Performance of proposed controller to stabilize the quadrotor and maintain the pitch angle to zero

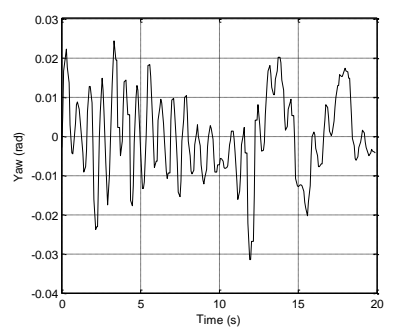

Figure 11. Performance of conventional backstepping controller to stabilize the quadrotor and maintain the yaw angle to zero

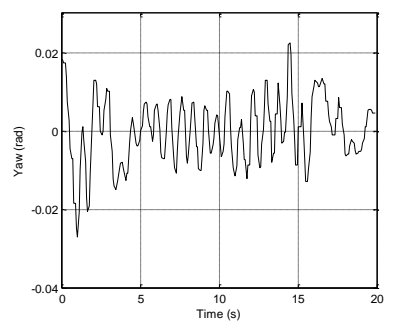

Figure 12. Performance of proposed controller to stabilize the quadrotor and maintain the yaw angle to zero

Performances of conventional backstepping controller and proposed backstepping controller considered nonlinear dynamics of motors are compared. Experiment results can be seen in Fig .7 - Fig .12.

It is difficult to give the same initial angular and angular speed to the roll and pitch angle in real flight experiments. The total performances in 20 seconds are compared between conventional backstepping controller and proposed controller. It can be seen in Fig .7 - Fig .12 that, the proposed controller has stronger ability to stabilize the vehicle. This is evidenced by the smaller error bound of roll and pitch angles' stabilization.

\section{CONCLUSIONS}

In this paper, a new method with respect to the improvement of the attitude stabilization for quadrotor helicopters is proposed. The major difference between current work and past ones is the use of nonlinear model of drive system in control strategy. By modeling brushless motor using second order Hammerstein series model, the accurate control of thrust is achieved. Both simulations and experiments on real system show that, with the same control law, proposed control strategy can achieve higher performance compared to ones without considering the motor dynamics. In future works, a more robust control algorithm would be taken into account to obtain better flight performances in the presence of disturbances

\section{REFERENCES}

[1] P. McKerrow, "Modelling the Draganflyer four-rotor helicopter", in Proceeding of IEEE International Conference on Robotics and Automation, 2004, pp. 3596-3601

[2] A. R. Patel, M. A. Patel and D. R. Vyas, "Modeling and analysis of quadrotor using sliding mode control", in Proceeding of 44th Southeastern Symposium on System Theory, 2012, pp. 111-114.

[3] R. Zhang, Q. Quan and K. Y. Cai, "Attitude control of a quadrotor aircraft subject to a class of time-varying disturbances", IET Control Theory and Applications, Vol. 5, No. 9, 2011, pp. 11401146.

[4] L. Besnard, Y. B. Shtessel and B. Landrum, "Quadrotor vehicle control via sliding mode controller driven by sliding mode disturbance observer", Journal of the Franklin Institute, Vol. 349, 2012, pp. 658-684.

[5] M. Kerma, A. Mokhtari and B. Abdelaziz, et al., "Nonlinear $\mathrm{H} \infty$ control of a Quadrotor (UAV), using high order sliding mode disturbance estimator", International Journal of Control, Vol. 85, No. 12, 2012, pp. 1876-1885.

[6] C. Nicol, C. J. Macnab and A. Ramirez-Serrano, "Robust adaptive control of a quadrotor helicopter", Mechatronics, Vol. 21, No. 6 2011, pp. 927-938.

[7] S. Bouabdallah, P. Murrieri and R. Siegwart, "Design and contro of an indoor micro quadrotor", in Proceeding of IEEE International Conference on Robotics and Automation, 2004, Vol. 5, pp. 4393 4398.

[8] P. Pounds, R. Mahony and P. Corke, "System identification and control of an aerobot drive system", in Proceeding of Information, Decision and Control, 2007, pp. 154-159.

[9] C. Djamila, M. Yahia and T. Ali, "Simultaneous Estimation of Rotor Speed and Stator Resistance in Sensorless Indirect Vector Control of Induction Motor Drives Using a Luenberger Observer", International Journal of Computer Science Issues, 2012, Vol. 9, No. 4, pp. 325-335.

[10] A. Manseur, D. Berkani and A. Mekhmoukh, "Adaptive filtering using Higher Order Statistics (HOS)", International Journal of Computer Science Issues, 2012, Vol. 9 No. 2, pp. 477-484.

[11] I. Fantoni and R. Lozano, Nonlinear control for underactuated mechanical systems, Springer, 2002 